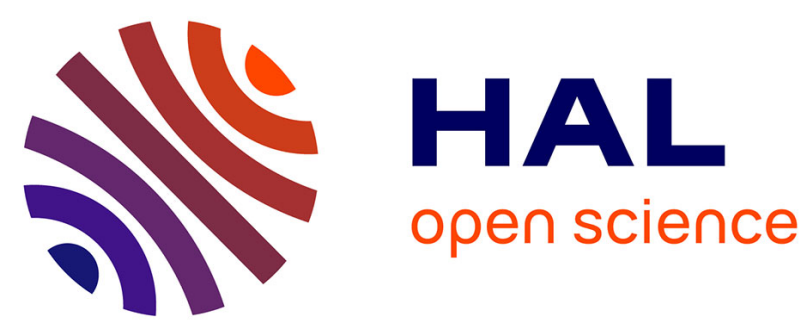

\title{
Design Parameters Influence on the Static Workspace and the Stiffness Range of a Tensegrity Mechanism
}

Giorgio Mackenzie Cruz-Martinez, Juan Carlos Avila-Vilchis, Adriana Vilchis González, Salih Abdelaziz, Philippe Poignet

\section{- To cite this version:}

Giorgio Mackenzie Cruz-Martinez, Juan Carlos Avila-Vilchis, Adriana Vilchis González, Salih Abdelaziz, Philippe Poignet. Design Parameters Influence on the Static Workspace and the Stiffness Range of a Tensegrity Mechanism. ARK 2020 - 17th International Symposium on Advances in Robot Kinematics, Dec 2020, Ljubljana, Slovenia. pp.15-24, 10.1007/978-3-030-50975-0_3 . lirmm-03141529

\section{HAL Id: lirmm-03141529 \\ https://hal-lirmm.ccsd.cnrs.fr/lirmm-03141529}

Submitted on 15 Feb 2021

HAL is a multi-disciplinary open access archive for the deposit and dissemination of scientific research documents, whether they are published or not. The documents may come from teaching and research institutions in France or abroad, or from public or private research centers.
L'archive ouverte pluridisciplinaire HAL, est destinée au dépôt et à la diffusion de documents scientifiques de niveau recherche, publiés ou non, émanant des établissements d'enseignement et de recherche français ou étrangers, des laboratoires publics ou privés. 


\title{
Design parameters influence on the static workspace and the stiffness range of a tensegrity mechanism
}

\author{
G. M. Cruz-Martinez ${ }^{1}$, J-C Avila Vilchis ${ }^{2}$, A. Vilchis Gonzalez ${ }^{2}$, S. Abdelaziz ${ }^{1}$ \\ and P. Poignet ${ }^{1}$
}

\begin{abstract}
This paper deals with the impact of the design parameters on the static workspace and the stiffness range of a planar 3-DoF tensegrity mechanism. The static model is established through the energetic approach and the stiffness is derived analytically along the $3-\mathrm{DoF}$ of the mechanism. The design parameters considered here are the spring stiffness and the location of the mechanism attachment points to the base. Results on the impact of these parameters are finally analyzed. This analysis constitutes a first step towards the geometric optimization of tensegrity mechanisms.
\end{abstract}

Key words: Tensegrity mechanism, static workspace, stiffness range.

\section{Introduction}

The term tensegrity was created by Richard B. Fuller as a union of 'tensional' and 'integrity' [6]. A tensegrity structure is formed entirely by a combination of rigid and flexible elements. Its configuration stands by itself and maintains its form solely because its structural members (struts) are suspended in a network of tensional elements (cables or springs) [13]. Tensegrity structures are characterized by being light, deployable and of variable stiffness [1]. Working with these structures has been of interest for engineers and researchers since tensegrity applications range from mobile robotics [5], manipulators [7, 14] and robots in medical applications [3].

Developing mathematical models (a kinematic one, for instance) for tensegrity mechanisms is challenging $[11,12,18]$ and requires to know the extension of flexible elements at all time so as to establish a relationship between the joint and the

The Authors (1) are with the LIRMM, Université de Montpellier, CNRS, France.

e-mail: [giorgio-mackenzie.cruz-martinez, abdelaziz, poignet]@lirmm.fr

The Authors (2) are with Autonomous University of Mexico State, Mexico.

e-mail: [avilchisg, jcavilav]@uaemex.mx 
cartesian variables. If the measurement of these extensions is not available, static modeling is considered. Several methods have been proposed in the literature to determine the equilibrium configurations of tensegrity mechanisms $[8,15]$. Static workspace computation, using a continuous approach, has been proposed by [2] for a 2-DoF tensegrity mechanism.

For the tensegrity mechanism reported in this paper, a potential energy approach is considered in order to determine stable equilibrium configurations based on the stiffness analysis. This paper is an attempt to understand the influence of one geometric parameter on static workspace and the stiffness range. The geometric parameter under observation concerns the mechanism attachment points. This analysis constitutes the first step towards the geometric optimization of tensegrity mechanisms.

This paper is organized as follow. The 3-DoF planar tensegrity mechanism is described in section 2 . The respective static and stiffness models are presented in subsection 2.1. These models are synthesized using an energetic approach. The computation of the static workspace and stiffness range are presented in subsection 2.2. The impact of the design parameters on the static workspace and the stiffness range is finally discussed in section 3 .

\section{Mechanism Description}

Fig 1 shows a 1-bar planar tensegrity mechanism driven by 4 actuators. According to the classification proposed by Skelton [17], this mechanism is a class-1 tensegrity system. The actuators are connected to the bar, of length $2 b$, using cables and springs. The springs are considered here identical of stiffnesses $k$. The cables, attached to the bar on $p_{1}$, are enrolled on pulleys fixed on $A_{1}$ and $A_{2}$ and are connected to springs before being enrolled on pulleys mounted on the actuators 1 and 2 . The other two cables, attached to $p_{2}$, are enrolled on pulleys fixed on $A_{3}$ and $A_{4}$ and are enrolled on pulleys mounted on the actuators 3 and 4 . 


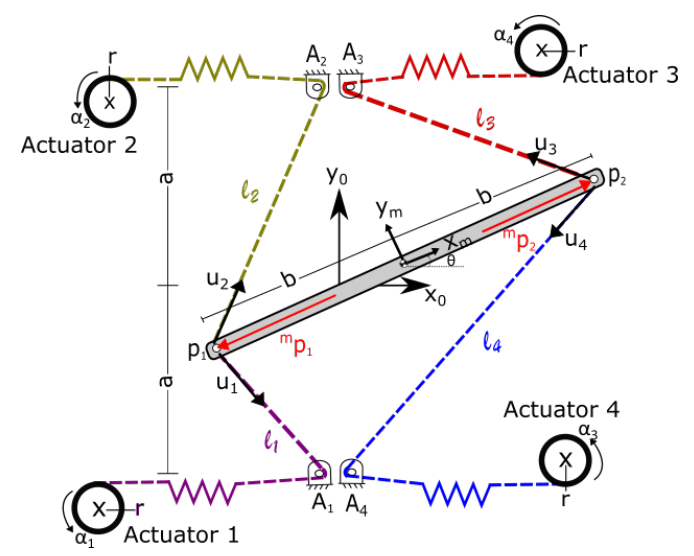

Fig. 1 Planar tensegrity mechanism

\subsection{Problem formulation}

As depicted in Fig. 1, the position of the center of mass $P$ of the bar is defined by $\mathbf{P}=[x, y]^{T}$ in the reference frame $\mathscr{R}_{0}=\left(O, \mathbf{x}_{0}, \mathbf{y}_{0}\right)$. A mobile frame $\mathscr{R}_{m}=\left(O_{m}, \mathbf{x}_{m}, \mathbf{y}_{m}\right)$, is attached to the bar's center of mass. The angle $\theta$ defines the bar's orientation. The coordinates of four attachment points $A_{1}, A_{2}, A_{3}$ and $A_{4}$ are defined, respectively, with respect to the inertial reference frame by position vectors $\mathbf{a}_{1}=[0,-a]^{T}, \mathbf{a}_{2}=[0, a]^{T}, \mathbf{a}_{3}=[0, n a]^{T}, \mathbf{a}_{4}=[0,-n a]^{T}$, with $n$-factor allowing to change locations of attachment points $A_{3}$ and $A_{4}$ where $0.1 \leq n<1$.

The coordinates of nodes $p_{1}$ and $p_{2}$ are defined with respect to the mobile frame $\mathscr{R}_{m}=\left(O_{m}, \mathbf{x}_{m}, \mathbf{y}_{m}\right)$ by vectors ${ }^{m} \mathbf{p}_{1}=[-b, 0]^{T}{ }^{m} \mathbf{p}_{2}=[b, 0]^{T}$ while with respect to the inertial frame $\mathscr{R}_{0}=\left(O, \mathbf{x}_{0}, \mathbf{y}_{0}\right)$ their coordinates are defined by equation 1 with $i=1,2$.

$$
\mathbf{p}_{i}=\mathbf{P}+\mathbf{R}_{m}{ }^{m} \mathbf{p}_{i}
$$

with $\mathbf{R}_{m}=\left(\begin{array}{cc}\cos \theta & -\sin \theta \\ \sin \theta & \cos \theta\end{array}\right)$

Let's define $\ell_{1}=\left\|\mathbf{a}_{1}-\mathbf{p}_{1}\right\|, \ell_{2}=\left\|\mathbf{a}_{2}-\mathbf{p}_{1}\right\|, \ell_{3}=\left\|\mathbf{a}_{3}-\mathbf{p}_{2}\right\|$ and $\ell_{4}=\left\|\mathbf{a}_{4}-\mathbf{p}_{2}\right\|$ as the distances between the bar's attachment points and the different nodes.

The system initial configuration is defined when the position of the bar's center of mass coincides with the origin of the inertial reference frame and $\theta=0$. Initial tensions in the cables/springs have minima values. Initial lengths are determined according to equation 2 .

$$
\ell_{0 g}=\ell_{0(g+1)}= \begin{cases}\sqrt{a^{2}+b^{2}} & \mathrm{~g}=1 \text { for cables } 1 \text { and } 2 \\ \sqrt{(n a)^{2}+b^{2}} & \mathrm{~g}=3 \text { for cables } 3 \text { and } 4\end{cases}
$$


Due to the presence of springs and in order to determine the static configuration of the mechanism, an energetic approach is considered. It means that the first and second derivatives of the potential energy of the system $U$ are used to establish the stable static equilibrium points. Equation 3 defines the total potential energy.

$$
U=\sum_{h=1}^{4} \frac{1}{2} k e_{h}^{2}
$$

where $e_{h}$ is the elongation of the spring $h$ that can be computed as in equation 4 .

$$
e_{h}=\rho_{h}+\ell_{h}-\ell_{0 h} \quad \text { for } \mathrm{h}=1,2,3,4
$$

with $\rho_{h}$ being the displacement of the cable $h$. It is computed as $\rho_{h}=r \alpha_{h}$, where $r$ is the radius of the actuator's pulley and $\alpha_{h}$ is the angular position of the actuator $h$.

The static equilibrium of the bar is obtained by, simultaneously, solving the equations 5 .

$$
\frac{\partial U}{\partial x}=0, \quad \frac{\partial U}{\partial y}=0, \quad \frac{\partial U}{\partial \theta}=0
$$

A stable equilibrium configuration must satisfy inequalities in equation 6.

$$
\frac{\partial^{2} U}{\partial x^{2}}>0, \quad \frac{\partial^{2} U}{\partial y^{2}}>0, \quad \frac{\partial^{2} U}{\partial \theta^{2}}>0
$$

\subsection{Characterization of the Static workspace and the Stiffness range}

The static workspace of a tensegrity system is defined as the set of all stable equilibrium configurations that its end-effector is able to reach [2], while taking into account the unilateral nature of the cables, i.e. The tensions in all the cables must remain positive.

Each point in the workspace has a minimum and maximum stiffness value. The range of stiffness for each point is computed by subtracting the maximum and the minimum stiffness values. In order to analyze the static workspace as well as the stiffness range of a tensegrity mechanism, as a function of design parameters, the tensions limits are considered identical in all the study.

The static equilibrium of the mechanism can be expressed in a similar way as for cable-driven parallel manipulators $[9,10]$ in accordance with equation 7

$$
\mathbf{W} \tau=\mathbf{f}
$$

where $\mathbf{W}$ represents a $3 \times 4$ wrench matrix that depends on the mechanism position $\mathbf{P}$ and its orientation $\theta$. The tensions in the cables are identified by vector $\tau=\left[\tau_{1}, \tau_{2}, \tau_{3}, \tau_{4}\right]^{T}$. The vector $\mathbf{f}$ represents the wrench applied to the mechanism by means of the cables tensions. The wrench matrix $\mathbf{W}$ is computed by Equation 8. 


$$
\mathbf{W}=\left[\begin{array}{cccc}
\mathbf{u}_{1} & \mathbf{u}_{2} & \mathbf{u}_{3} & \mathbf{u}_{4} \\
\mathbf{p}_{1} \times \mathbf{u}_{1} & \mathbf{p}_{1} \times \mathbf{u}_{2} & \mathbf{p}_{2} \times \mathbf{u}_{3} & \mathbf{p}_{2} \times \mathbf{u}_{4}
\end{array}\right]
$$

where $\mathbf{u}_{h}$ represents the unit vector. Its direction is defined as the cable direction (cf. Fig 1).

The tension in the cables must remain in between a minimum and a maximum values, respectively $\tau_{\text {min }}$ and $\tau_{\text {max }}$. The stiffness range for a given stable equilibrium configuration is computed by varying the tensions in the cables without affecting the position and orientation of the mechanism. These tensions are computed as:

$$
\tau=\mathbf{W}^{+} \mathbf{f}+\mathbf{H} \lambda
$$

where $\boldsymbol{W}^{+}$is the Moore-Penrose generalized inverse of $\mathbf{W}$ [16]. $\mathbf{H}$ is a vector of dimension $4 \times 1$ whose column span the null space of $\mathbf{W}$. $\lambda$ is an arbitrary scalar. To keep a stable equilibrium configuration, the wrench $\mathbf{f}$ is considered equal to a null vector. The bounds of $\lambda$ can be obtained by solving the inequality 10 .

$$
\tau_{\min } \leq \mathbf{H} \lambda \leq \tau_{\max }
$$

The bounds $\lambda_{\min }$ and $\lambda_{\max }$ allowing to compute two vector tensions solutions that enable to keep the equilibrium:

$$
\begin{aligned}
\tau_{\min } & =\mathbf{H} \lambda_{\min } \\
\tau_{\max } & =\mathbf{H} \lambda_{\max }
\end{aligned}
$$

Using the vectors $\tau_{\min }$ and $\tau_{\max }$, one can compute the minimum and maximum springs elongations. Solving the equation 4 , can be calculated $\rho^{\min }$ and $\rho^{\max }$ both are vectors that contain the set of minimum and maximum displacements for each actuators. Now the minimum and maximum stiffness are computed using the equation 12 at a given pose of the mechanism $(\mathbf{P}, \theta)$ considering $\rho^{\min }$ and $\rho^{\max }$.

$$
\begin{aligned}
& K_{x}^{\text {min }}=\frac{\partial^{2} U\left(\mathbf{P}, \theta, \rho^{\text {min }}\right)}{\partial x^{2}}, \quad K_{y}^{\text {min }}=\frac{\partial^{2} U\left(\mathbf{P}, \theta, \rho^{\text {min }}\right)}{\partial y^{2}}, \quad K_{\theta}^{\text {min }}=\frac{\partial^{2} U\left(\mathbf{P}, \theta, \rho^{\text {min }}\right)}{\partial \theta^{2}} \\
& K_{x}^{\text {max }}=\frac{\partial^{2} U\left(\mathbf{P}, \theta, \rho^{\text {max }}\right)}{\partial x^{2}}, \quad K_{y}^{\text {max }}=\frac{\partial^{2} U\left(\mathbf{P}, \theta, \rho^{\text {max }}\right)}{\partial y^{2}}, \quad K_{\theta}^{\text {max }}=\frac{\partial^{2} U\left(\mathbf{P}, \theta, \rho^{\text {max }}\right)}{\partial \theta^{2}}
\end{aligned}
$$

\section{Discussion of the results}

This section show the estimation of the static workspace and stiffness range. The static workspace of the system describes the pose of the end-effector in the $\mathrm{x}, \mathrm{y}$ coordinates and the orientation along $\mathrm{z}$ axis. The parameters of the mechanism that are used were: $\mathrm{a}=0.1 \mathrm{~m}, \mathrm{~b}=0.1 \mathrm{~m}, \tau_{h} \in[4,10] \mathrm{N}$ and $\rho_{h} \in\left[\frac{4}{k}, \frac{10}{k}\right] \mathrm{m}$. 
The Fig. 2 shows the relationship between the size of the workspace and the spring stiffness values $k$. The first column shows the static workspace of the mechanism with $n=1$ and $k=270 \mathrm{~N} / \mathrm{m}$ and the second column shows the static workspace when $n=1$ and $k=80 \mathrm{~N} / \mathrm{m}$. The black dots represent the end-effector in a stable equilibrium configuration that the mechanism can reach, the red line shows the mechanism's boundary of static workspace with $k=270 \mathrm{~N} / \mathrm{m}$. In the second column the green line shows the boundary of static workspace with $k=80$ $\mathrm{N} / \mathrm{m}$ and the red line is superimposed to highlight how the workspace is affected in relationship with the modification of the stiffness springs, noting that the value of $k$ is inversely proportional to the static workspace.
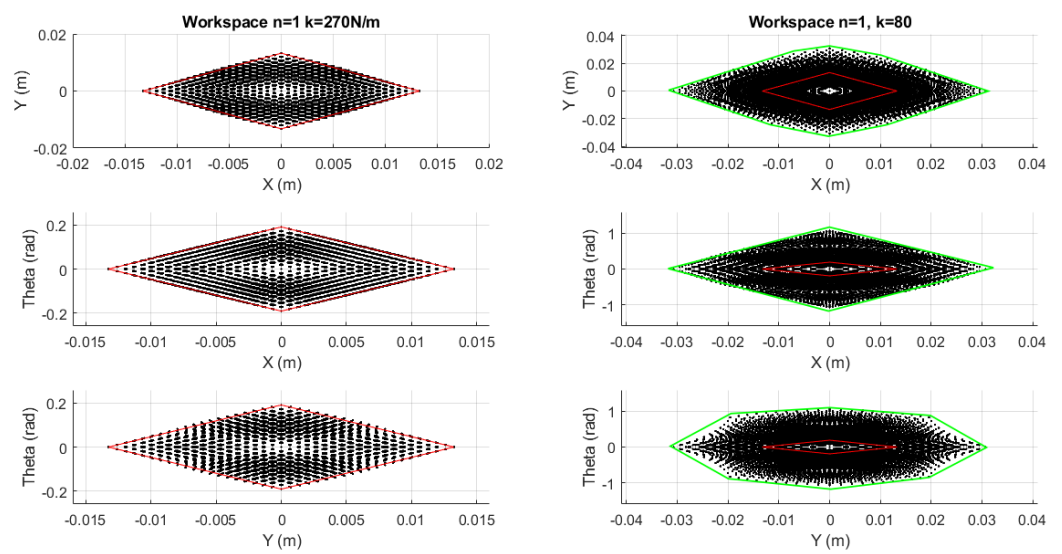

Fig. 2 Relationship between static workspace and variation of $\mathrm{k}$

In order to analysis the influence of the geometrical parameters on the static workspace and stiffness range, here is presented the results when $n=1$ (Fig. 1), $n=0.5$ (Fig.3a) and $n=0.1$ (Fig. 3b). These variation of $n$ causes that the symmetry in the y-axis be lost because the nodes $A_{3}, A_{4}$ reducing the distance between them. 

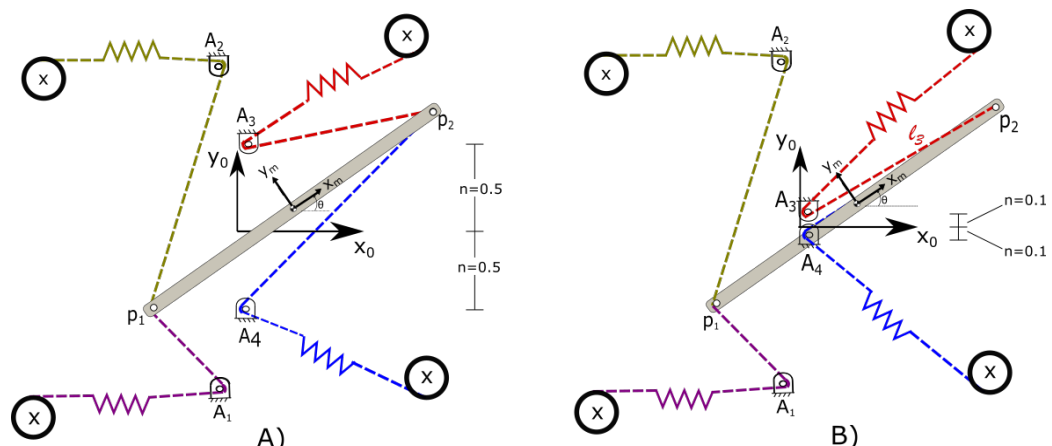

Fig. 3 A) Tensegrity mechanism with $n=0.5$, B) Tensegrity mechanism with $n=0.1$

The Fig. 4 illustrates the static workspace for the mechanism when $n=1,0.5$ and 0.1 . The workspace for $n=1$ has ranges from $x \in(-0.2,0.2) m, y \in(-0.2,0.2) m$ and $\theta \in(-1.5,1.5) \mathrm{rad}$. The shape is modified throughout the changes of $n$, this changes are described by the projections on the planes $x y, y \theta, x \theta$. The black dots represent the center of mass in a stable equilibrium configuration that the mechanism can reach, the red line shows the mechanism's boundary of static workspace with $n=1$ and the green line shows the boundary of static workspace for $n=0.5$ and $n=0.1$ respectively. As the Fig. 4 pictures the workspace in $x y$ is decreased as $n$ decreases and is shifted to the other side where the attachment points joint. However for the workspace $x \theta$ who is showed in row 2 increases as $n$ decreases. The largest workspace in the plane $y \theta$ is in the configuration with $n=0.5$.
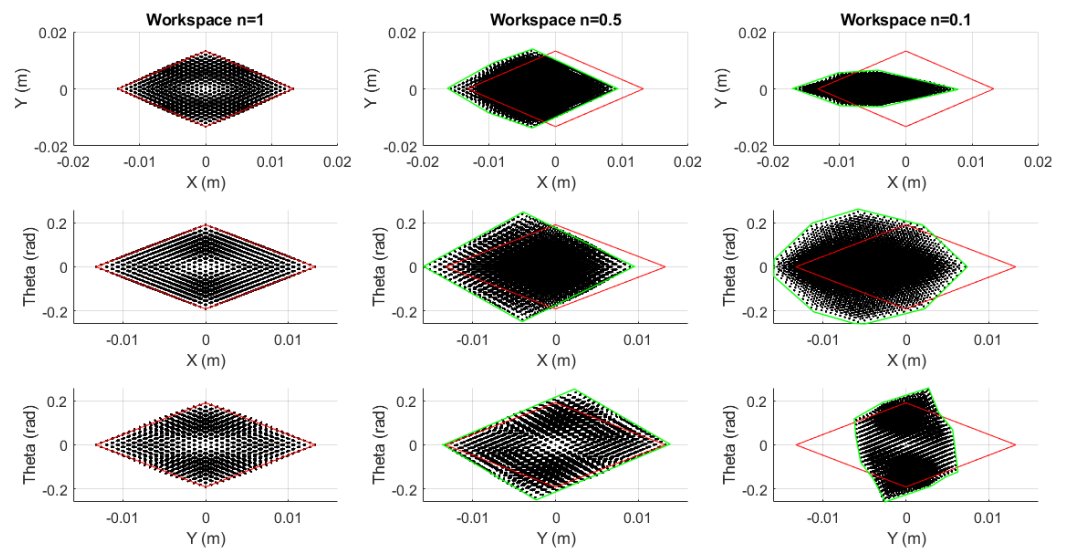

Fig. 4 Static workspace when $n=1,0.5$ and 0.1

The analysis of the stiffness range is shown below. The Fig. 5 shows the stiffness range for $K_{x}$, the Fig. 6 shows the stiffness range for $K_{y}$ and the Fig. 7 shows the 
stiffness range for $K_{\theta}$. The workspace is depicted as a volume. In order to observe the behavior of stiffness, it has been discretized in layers. The first column of each figure represents the minimum stiffness and the second column shows the stiffness range, each point in the workspace has a color according to its stiffness value. The black line represents the boundary of the workspace with $n=1$ and it is superimposed on all the graphs to show how the workspace changes.
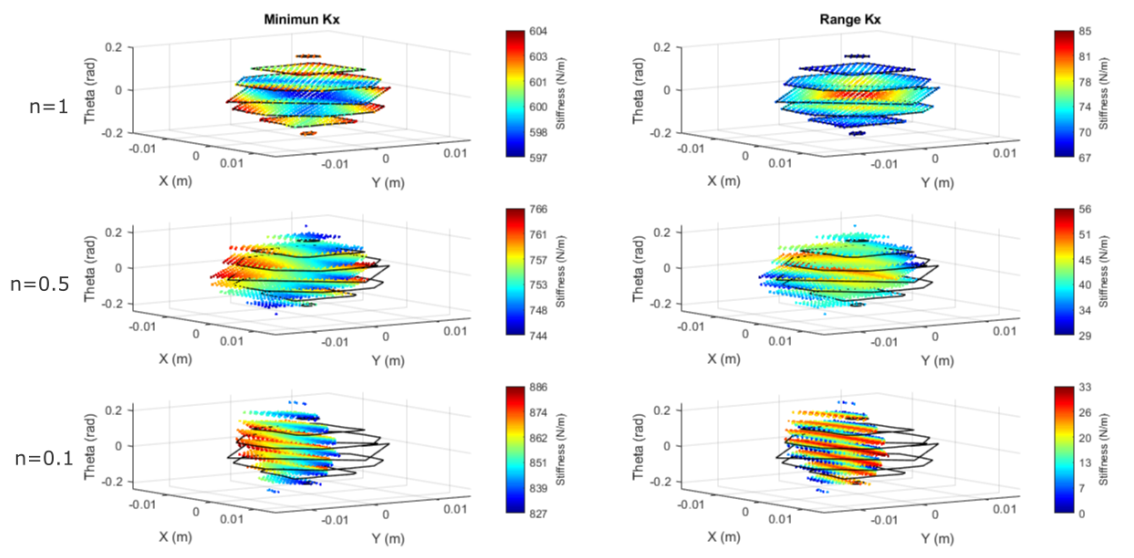

Fig. 5 The stiffness ranges $K_{x} n=1,0.5$ and 0.1
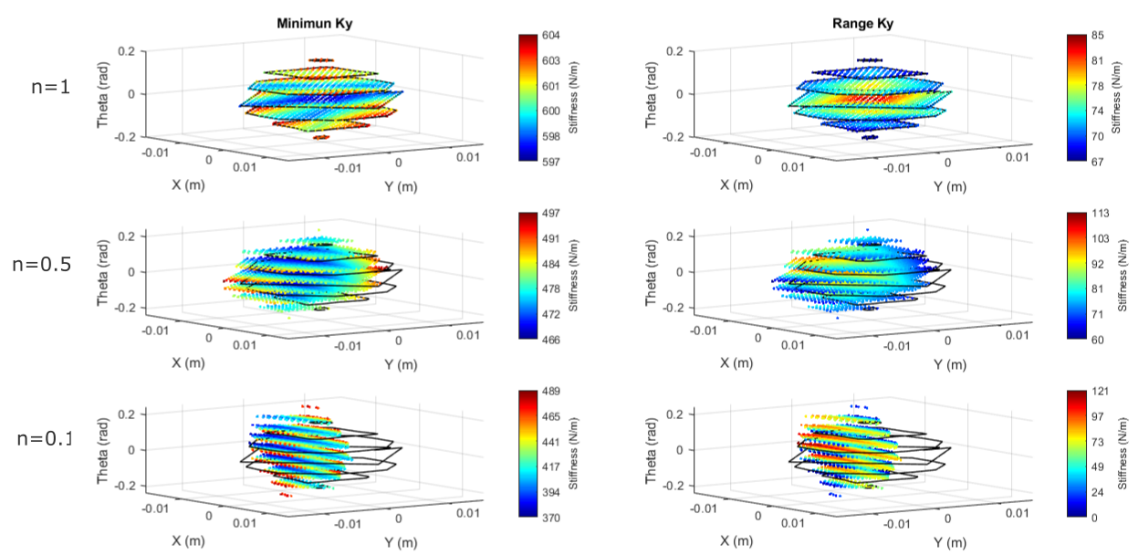

Fig. 6 The stiffness ranges $K_{y}$ with $n=1,0.5$ and 0.1 

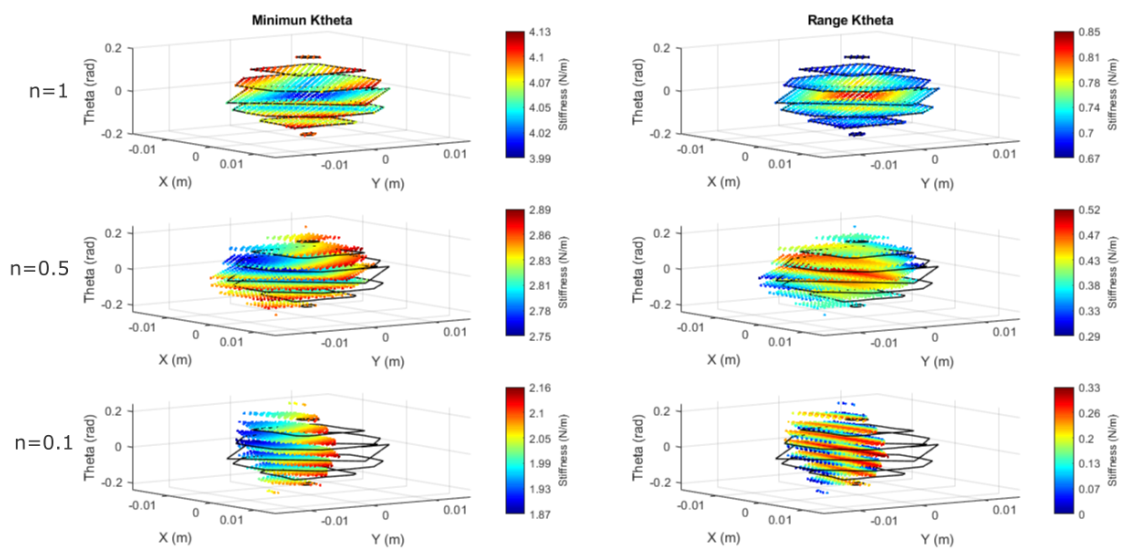

Fig. 7 The angular stiffness ranges $K_{\theta}$ with $n=1,0.5$ and 0.1

This tensegrity mechanism could be used as a puncture assistance robot $[3,4]$ because it allows stiffness modulation. The procedure requires that the effector has to be rigid when a needle is inserted in the body and in other moment it has to be soft allowing to follow the physiological movements such as breath.

To illustrate the application of the computation of the figures 5, 6 and 7, two desired stiffness ranges are selected according to a specific application: $K_{x} \geq 30 \mathrm{~N} / \mathrm{m}$ and $K_{y} \geq 75 \mathrm{~N} / \mathrm{m}$. The Fig. 8 illustrates the workspace that satisfies the desired stiffness range, the first row shows $K_{x}$ with $n=1,0.5$ and 0.1 and the second row shows the workspace for $K_{y}$ desired with $n=1,0.5$ and 0.1 . The workspace for the range $K_{x}$ is greater with $n=0.5$ since it allows reach higher orientation values. The same behavior happens in the workspace for the range $K_{y}$ but the increase is more notorious in the mobility orientation with $n=0.5$. Concluding that the biggest workspace that satisfies the design conditions for the application is with the geometrical parameter $n=0.5$. 

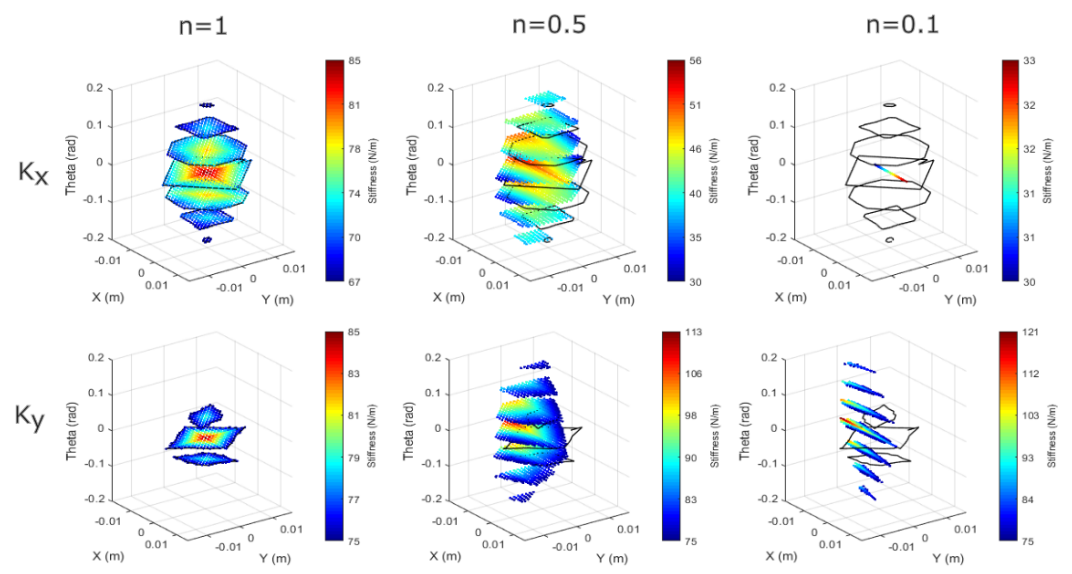

Fig. 8 Workspace using $K_{x}=30 \mathrm{~N} / \mathrm{m}$ and $K_{y}=75 \mathrm{~N} / \mathrm{m}$

\section{Conclusion}

This paper shows the influence of the design parameters on the static workspace as well as in the stiffness range of a 3-DoF planar tensegrity mechanism. An extension of the approach analysis can be applied for tensegrity mechanism with more degrees of freedom. The analysis will help to design a geometric optimization approach that allows to define the location of the attachment points and to select the adequate springs in order to satisfy a required static workspace with a desired stiffness range.

\section{References}

1. Azadi, M., Behzadipour, S., Faulkner, G.: Variable stiffness spring using tensegrity prisms. Journal of Mechanisms Robotics ASME (2010). DOI 10.1115/1.4001776

2. Boehler, Q., Charpentier, I., Vedrines, M.S., Renaud, P.: Definition and computation of tensegrity mechanism workspace. Journal of Mechanisms and Robotics (2015). DOI $10.1115 / 1.4029809$

3. Boehler, Q., Zompas, A., Vedrines, M., Abdelaziz, S., Renaud, P., Poignet, P.: Experiments on a variable stiffness tensegrity mechanism for an mr-compatible needle holder. Computer/Robot Assisted Surgery (2015)

4. Bricault, I., Jauniaux, E., Zemiti, N., Fouard, C., Taillant, E., Dorandeu, F., Cinquin, P.: Light puncture robot for ct and mri interventions. IEEE Engineering in Medicine and Biology Magazine 27 (2008). DOI 10.1109/EMB.2007.910262

5. Friesen, J., Pogue, A., Bewley, T., De Oliveira, M., Skelton Robert andl Vytas, S.: Ductt: a tensegrity robot for exploring duct systems. IEEE International Conference on Robotics and Automation (ICRA) (2014). DOI 10.1109/IROS.2016.7759811

6. Fuller, B. (ed.): Synergetics, explorations in the geometry of thinking. Collier Macmillan (1975) 
7. Furet, M., Chablat, D., Fasquelle, B., Khanna, P., Chevallereau, C., Wenger, P.: Prototype of a tensegrity manipulator to mimic bird necks. In: 24ème Congrès Français de Mécanique. Brest, France (2019)

8. Furet, M., Wenger, P.: Kinetostatic analysis and actuation strategy of a planar tensegrity $2-\mathrm{x}$ manipulator. Journal of Mechanisms and Robotics (2019). DOI 10.1115/1.4044209

9. Gagliardini, L., Gouttefarde, M., Caro, S.: Determination of a dynamic feasible workspace for cable-driven parallel robots. Advances in Robot Kinematics 2016, Springer Proceedings in Advanced Robotics, (2017)

10. Gouttefarde, M., Merlet, J.P., Daney, D.: Wrench-feasible workspace of parallel cable-driven mechanisms. In: IEEE International Conference on Robotics and Automation. Roma, Italy (2007). DOI 10.1109/ROBOT.2007.363195

11. Henrickson, J.V., Valaseky, J., Skelton, R.: Shape control of tensegrity structures. In: AIAA SPACE 2015 Conference and Exposition. Pasadena, USA (2015). DOI 10.2514/6.2015-4502

12. Ji, Z., Li, T., Lin, M.: Kinematics, singularity, and workspaces of a planar 4-bar tensegrity mechanism. Journal of Robotics (2014). DOI 10.1155/2014/967251

13. Jing, Y.Z., Ohsaki, M. (eds.): Tensegrity Structures Form, Stability and Symmetry. Springer (2015). DOI 10.1007/978-4-431-54813-3

14. Lessardand, S., Castro, D., Asper, W., Chopra, S.D., Baltaxe-Admony, L., Teodorescu, M., SunSpiral, V., Agogino, A.: A bio-inspired tensegrity manipulator with multi-dof, structurally compliant joints. In: 2016 IEEE/RSJ International Conference on Intelligent Robots and Systems (IROS), pp. 5515-5520 (2016). DOI 10.1109/IROS.2016.7759811

15. Manríquez-Padilla, C.G., Zavala-Pérez, O.A., Pérez-Soto, G.I., Rodríguez-Reséndiz, J., Camarillo-Gómez, K.A.: Form-finding analysis of a class 2 tensegrity robot. Applied sciences (2019). DOI 10.3390/app9152948

16. Roberts, R.G., Graham, T., Lippitt, T.: On the inverse kinematics, statics, and fault tolerance of cable-suspended robots. Journal of Robotic Systems (1998). DOI 10.1002/(SICI)10974563(199810)15:10;581::AID-ROB4¿3.0.CO;2-P

17. Skelton, R., Adhikari, R., Pinaud, J.P., Chan, W.: An introduction to the mechanics of tensegrity structures. In: Conference on Decision and Control. Florida, USA (2001). DOI $10.1109 / .2001 .98086$

18. Wenger, P., Chablat, D.C.: Kinetostatic analysis and solution classification of a planar tensegrity mechanism. In: Computational Kinematics, pp. 422-431. Springer International Publishing (2018). DOI 10.1007/978-3-319-60867-948 